\title{
AVALIAÇÃO DO PERÍODO DE FORNECIMENTO DE ANTIBIÓTICO COMO PROMOTOR DE CRESCIMENTO SOBRE O DESEMPENHO DE FRANGOS DE CORTE
}

Éder Clementino dos Santos - Instituto Federal de Educação, Ciência e Tecnologia Sul de Minas Gerais - Campus de Inconfidentes - www.eafi.gov.br - ederclementino@eafi.gov.br, Elesandre Clementino dos Santos

\section{RESUMO}

Com o objetivo de avaliar o período de fornecimento de antibiótico como promotor de crescimento sobre o desempenho dos frangos de corte no período de 1 a 42 dias de idade criados em cama reutilizada, foi realizado um experimento no Departamento de Zootecnia da Universidade Federal de Lavras-UFLA/MG. Foram avaliados o consumo de ração, o ganho de peso e a conversão alimentar. As aves foram alimentadas com ração à base de milho e farelo de soja, suplementadas com dois tipos de antibióticos em um delineamento inteiramente casualizado em esquema fatorial $5 \times 2 \times$ 2 (período de retirada do antibiótico x antibiótico x sexo) com 10 tratamentos, 3 repetições, 25 aves por parcela experimental. Adição de avilamicina e bacitracina de zinco apresentou benefícios no desempenho das aves nesse período de criação. Os resultados demonstraram haver efeito dos tratamentos estudados para consumo de ração, ganho de peso, conversão alimentar, proporcionando melhor desempenho às aves machos que receberam antibióticos somente até aos 20 dias de idade em relação àquelas que tiveram uso contínuo dos mesmos.

TERMOS PARA INDEXAÇÃO: Frango de corte, antibiótico, desempenho, conversão alimentar.

\section{EVALUATION OF PERIOD OF THE SUPPLY OF THE ANTIBIOTIC AS PROMOTER OF GROWTH UNDER PERFORMANCE OF BROILERS CHICKENS}

\begin{abstract}
The objective of this work was to evaluate the effect of the period of the supply of antibiotic as growth promoter under the performance of broiler chickens raised in the period of 1 to 42 days of age with old litter. The experimet was conducted at the Animal Science Departament of the Federal University of Lavras. The parameters evaluated were ration consumption, body weigth gain and food conversion. The birds were fed with corn and soybean ration, with two types of antibiotics in a completely randomized experimental design in a $5 \times 2 \times 2$ factorial scheme (retreated period $\mathrm{x}$ antibiotic x sex) with 10 treatments, 3 replications, 25 birds for experimental unit. Avilamicin and bacitracin of zinc presented benefits in the performance of chickens. The data demonstrated that there is an effect of the treatments studied for ration consumption, body weigth gain, food conversion, providing better acting to the birds male that received antibiotics only until 20 days of age in relation to those that had continuous use of the same ones.
\end{abstract}

INDEX TERMS: Broiler chicken, antibiotic, performance, food conversion. 


\section{INTRODUÇÃO}

Os antibióticos são agentes antimicrobianos sintetizados por microorganismos vivos como leveduras, fungos e bactérias que podem ser usados em rações de animais em níveis subterapêuticos, favorecendo uma ação promotora do crescimento e no aumento na eficiência de produção, de forma que esses aditivos alimentares ainda são utilizados para melhorar o desempenho animal. Esse efeito promotor de crescimento pode ser influenciado pela composição da dieta, pelo ambiente como também por fatores genéticos (CHOI e RYU, 1987).

A tecnologia empregada no setor avícola tem proporcionado uma obtenção de alta produtividade, aliada à qualidade de um alimento mais seguro para o consumidor, que dentre os vários problemas encontrados na avicultura, a saúde do consumidor passou a ter maior importância na cadeia de produção animal. Várias pesquisas têm sido realizadas com o intuito de incrementar a utilização de aditivos alternativos e com menor custo para substituição dos antibióticos promotores de crescimento. Apesar dos grandes benefícios obtidos pelo uso de níveis subterapêuticos dos antibióticos na criação de frangos de corte, têm surgido críticas severas em relação ao uso rotineiro deste aditivo, por parte dos órgãos oficiais de saúde pública e da população de uma maneira geral, principalmente nos países da Europa (SPRING, 2000). No entanto, não há comprovação científica de que os antibióticos promotores de crescimento utilizados nas rações de aves, suínos e bovinos deixem resíduos nos alimentos que possam provocar impactos negativos à saúde humana (IAFIGLIOLA, 1999). A alternativa de retirada dos antibióticos da alimentação de frangos de corte através de forma periódica tem por objetivo reduzir seus níveis na ração e procurar manter as ações benéficas dos mesmos eliminando ações indesejáveis tais como indução do aparecimento de cepas resistentes às drogas, que pode causar outros danos ao consumidor. O objetivo desse traba- lho foi avaliar o período de fornecimento de antibiótico como promotor de crescimento sobre o desempenho dos frangos de corte criados no período de 1 a 42 dias de idade.

\section{MATERIAL E MÉTODOS}

O experimento foi desenvolvido no Setor de Zootecnia do Instituto Federal de Educação, Ciência e Tecnologia, Campus de Inconfidentes-MG. Foram utilizados 1500 pintos de corte da linhagem Ross no período de 1 a 42 dias de idade, distribuídos em um delineamento inteiramente casualizado com 10 tratamentos num esquema fatorial $5 \times 2 \times 2$ (período de fornecimento de antibiótico $\mathrm{x}$ antibiótico x sexo) com 3 repetições de 25 aves por parcela experimental. Os períodos de fornecimento de antibiótico foram aos 0,10,20,30 e 40 dias de idade, e os antibióticos avilamicina e bacitracina de zinco foram utilizados na proporção de 10 ppm e 50 ppm na ração, respectivamente. As dietas fornecidas foram à base de milho e farelo de soja com níveis de proteína bruta fixados em $21,5 \%$ ( 1 a 21 dias) e 19\% (22 a 42 dias) e os níveis de energia metabolizável fixados em $3000 \mathrm{Kcal}$ (1 a 21 dias) e $3100 \mathrm{Kcal}$ (22 a 42 dias), segundo recomendações de ROSTAGNO et al. (2000). As aves foram criadas em cama reutilizada com o objetivo de aumentar o poder de desafio aos promotores de crescimento. Ao final do período de 42 dias 04 (quatro) aves de cada parcela experimental (duas por sexo) foram pesadas para avaliação do ganho de peso, consumo de ração e conversão alimentar. As análises estatísticas foram realizadas pelo pacote computacional SISVAR (Sistema de Análise de Variância para dados Balanceados) segundo FERREIRA (1998), utilizando o modelo de regressão para período de fornecimento de antibióticos e para os fatores antibiótico e sexo as médias foram comparadas pelo teste de TUKEY a 5\% de probabilidade. 


\section{RESULTADOS E DISCUSSÃO}

Os resultados de consumo de ração, ganho de peso e conversão alimentar das aves no período de 1 a 42 dias de idade estão expressos no quadro 1 e nas figuras 1, 2 e 3, respectivamente. Nesse período não houve interação significativa entre consumo de ração e conversão alimentar, portanto, foram analisados separadamente. Para a variável consumo de ração houve diferenças significativas $(\mathrm{P}<0,01)$ somente para sexo. As aves machos tiveram maior consumo em média de 9,9\% em relação às fêmeas, independentemente dos tratamentos utiliza- dos. Pôde-se verificar, que embora não tenha havido diferenças significativas entre os tratamentos, as aves que receberam antibióticos até aos 20 dias de idade tiveram numericamete maior consumo em relação às aves que receberam ração sem antibióticos, assim como as aves que receberam dietas com antibióticos durante os 30 e 40 dias de idade comparadas às aves que receberam dietas sem antibióticos. Foi observado que o maior consumo de ração ocorreu aos 23 dias de idade (figura 1), e à medida em que ocorreu a permanência dos antibióticos nas dietas após essa idade, verificou-se uma redução no consumo em ambos os sexos.

Tabela 1 - Desempenho de frangos de corte submetidos a diferentes períodos de fornecimento de antibiótico, tipo de antibiótico e sexo no período de 1 a 42 dias de idade.

\begin{tabular}{lccc}
\hline $\begin{array}{l}\text { Período de } \\
\text { fornecimento (dias) }\end{array}$ & $\begin{array}{c}\text { Consumo } \\
\text { de ração }(\mathrm{g})\end{array}$ & $\begin{array}{c}\text { Ganho de } \\
\text { peso }(\mathrm{g})\end{array}$ & $\begin{array}{c}\text { Conversão } \\
\text { alimentar }^{1}\end{array}$ \\
\hline 0 & 3566 & 1952 & 1,82 \\
10 & 3640 & 2004 & 1,81 \\
20 & 3693 & 2008 & 1,83 \\
30 & 3652 & 1959 & 1,86 \\
40 & 3624 & 1898 & 1,90 \\
\hline Avilamicina & 3656 & 1971 & 1,85 \\
Bacitracina de zinco & 3614 & 1957 & 1,84 \\
\hline Macho & $3807 \mathbf{~ a ~}$ & $2063 \mathbf{~ a}$ & 1,84 \\
Fêmea & $3463 \mathbf{~ b}$ & $1865 \mathbf{~ b}$ & 1,85 \\
\hline CV $(\%)$ & 3,38 & 3,37 & 2,33 \\
\hline
\end{tabular}

${ }^{1}$ Efeito quadrático $-{ }^{2}$ Efeito linear

Médias seguidas de letras distintas, na coluna, diferem entre si pelo teste de Tukey $(\mathrm{P}<0,05)$

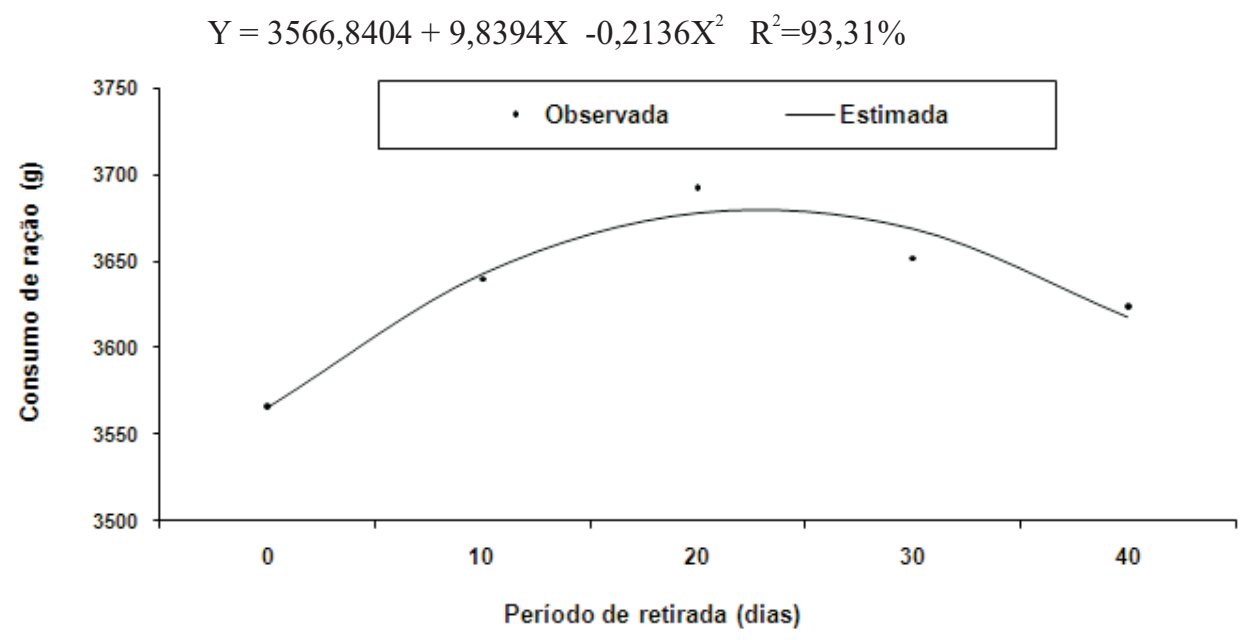

Figura 1- Consumo de ração (g) segundo os períodos de fornecimento de antibiótico 
Foi observado interação significativa do período de fornecimento de antibiótico e sexo $(\mathrm{P}<0,04)$ sobre o ganho de peso das aves. Os sexos influenciaram no desempenho dos frangos nesse período, onde as aves machos tiveram maior ganho de peso em média $10,62 \%$ do que as fêmeas (Figura 2). O ganho máximo ocorreu aos 19 dias de idade e reduziu após essa idade indicando um efeito quadrático $(\mathrm{P}<0,01)$. Já para as fêmeas, foi observado um efeito linear $(\mathrm{P}<0,01)$ independentemente dos tratamentos e verificou-se que a permanência dos antibióticos na ração deprimiu o ganho de peso das mesmas, sugerindo que são mais sensíveis à presença de antibióticos na dieta em relação ao machos. Com a presença dos antibióticos na dieta, possivelmente, os microrganismos que deveriam facilitar o cres- cimento das aves fêmeas podem ter estimulado aumento da viscosidade intestinal e, provavelmente, pode ter influenciado no processo digestão e absorção de nutrientes e como consequência, o ganho de peso foi afetado negativamente, apesar do bom manejo, boa qualidade da ração e da resposta ao desafio à cama reutilizada. Estes resultados não são condizentes com ALVARES et al. (1994), HENRIQUE et al. (1998) e DIONÍZIO (2001) que não encontraram diferenças nos tratamentos para consumo de ração e ganho de peso. No entanto, os resultados desse trabalho corroboram com aqueles obtidos por BERTECHINI e HOSSAIN (1993) e ZUANON et al. (1998) TEIXEIRA et al. (2002) que verificaram uma melhora nas variáveis supramencionadas.

$$
\begin{array}{ll}
\text { Macho } Y=2017,5619+11,4895 X-0,3061 X^{2} & R^{2}=85,60 \% \\
\text { Fêmea } Y=1911,0000-2,2800 X & R^{2}=66,31 \%
\end{array}
$$

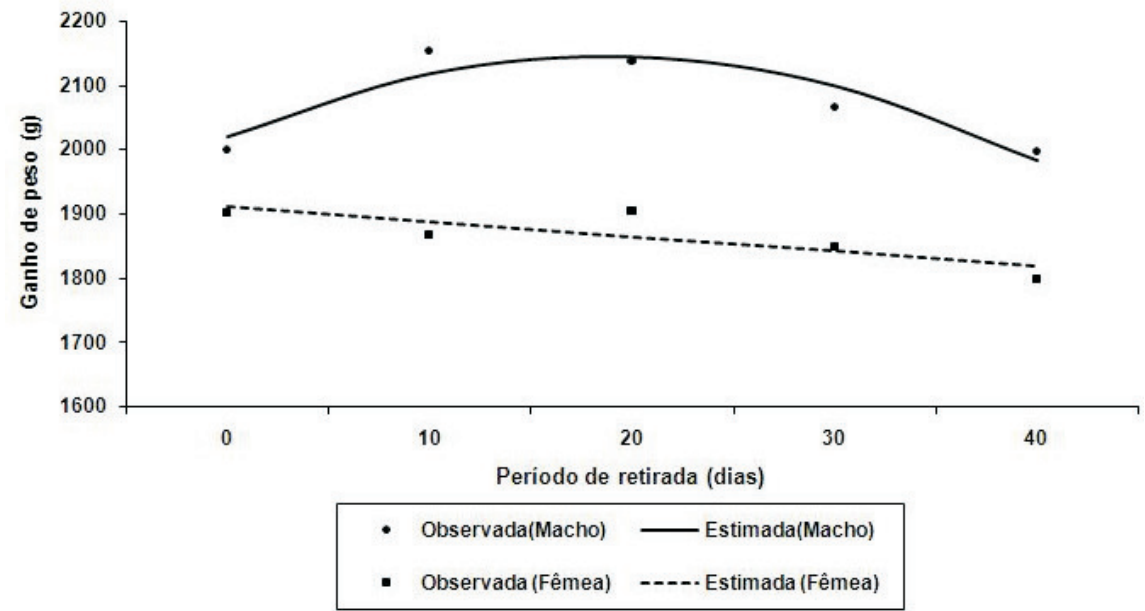

Figura 2 - Ganho de peso ( $\mathrm{g}$ ) em função dos períodos de fornecimento dos antibióticos

Para conversão alimentar verificou-se que somente houve diferença significativa $(\mathrm{P}<0,01)$ para o período de retirada de antibióticos das dietas.Nenhum dos fatores sexos e tipos de antibióticos não influenciaram na conversão alimentar (Figura 3). A não significância da conversão alimentar das aves possivelmente, deveu-se à redução do ganho de peso e do consumo de ração. A melhor conversão ocorreu aos 7 dias de idade sugerindo a não necessidade da permanência desses aditivos acima dessa idade para promover melhora no desempenho. Esses resultados estão de acordo com aqueles obtidos por PENSACK et al. (1982), ALVAREZ et al. (1994), KRINKE e JAMROZ (1996) e TEIXEIRA et al. (2002) que constataram efeito favorável nessa variável quando usou antibiótico na ração. Para o período total de criação de 1 a 42 dias de idade, as variáveis de desempenho apresentaram efeito benéfico quando do fornecimento dos antibióticos no 
período de inicial de 1 a 21 dias foi mantido para o período total de criação contrariando os resultados obtidos por HENRIQUE et al. (1997) e LODDI et al. (2000) que não obtiveram efeito significativo nesse período.
As diferenças entre sexo, superiores para os machos nas características de desempenho corroboram com os obtidos por BERTECHINI e HOSSAIN (1993), WOLKE et al. (1996) e ZUANON et al. (1998).

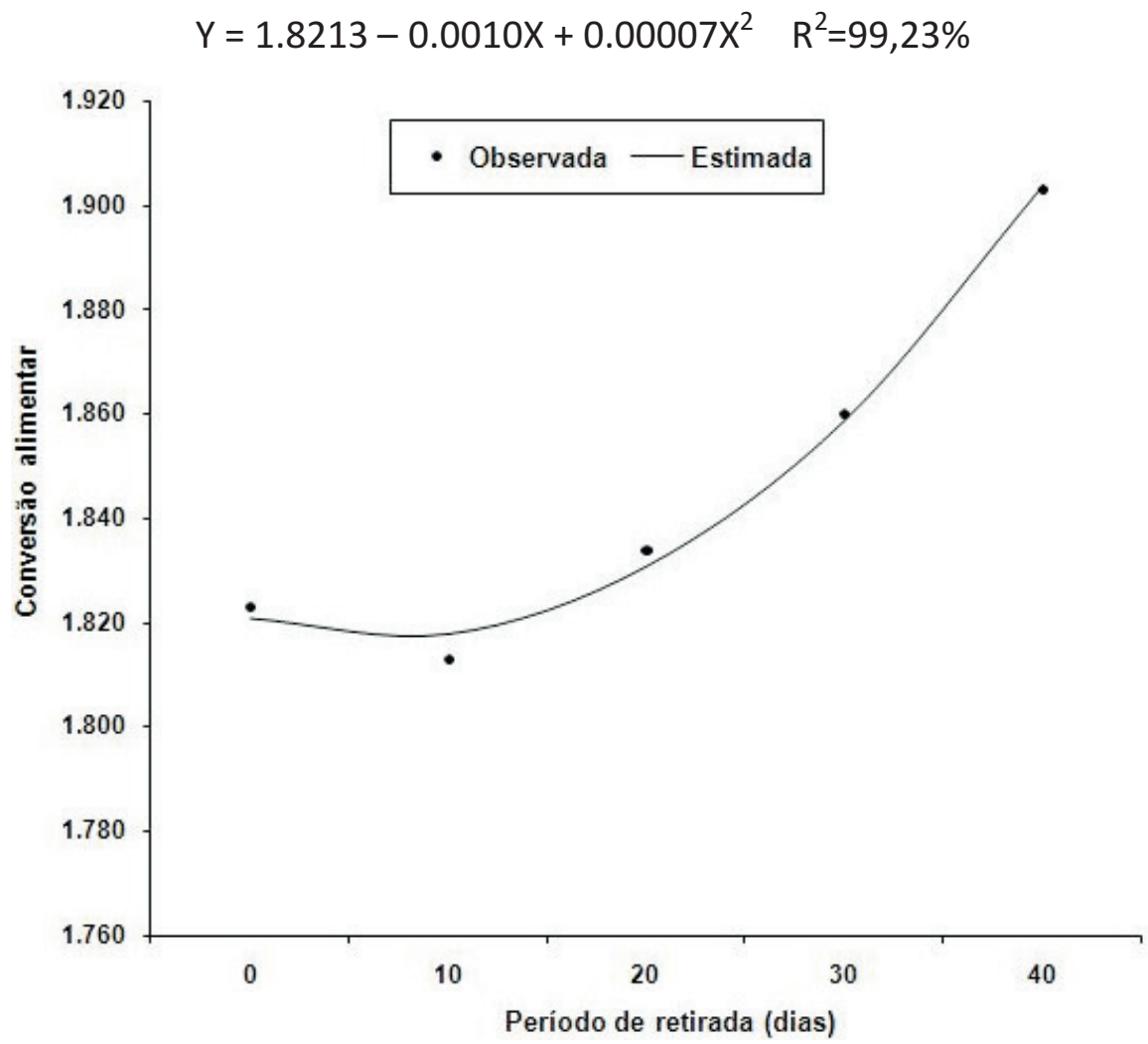

Figura 3 -Conversão alimentar em função dos períodos de fornecimento de antibióticos

\section{CONCLUSÕES}

Nas condições em que foi conduzido o experimento, o período de fornecimento de antibióticos como promotores de crescimento proporcionou melhor desempenho zootécnico às aves machos aos 42 dias de idade, indicando a não necessidade desses aditivos após o período da fase inicial e, consequentemente, possibilitou a redução desses antibióticos na ração de frangos de corte.

\section{REFERÊNCIAS BIBLIOGRÁFICAS}

ALVAREZ, L.C.; BARRERA, E.M.; GONZÁLES, E.A. Evaluación de promotores del crecimiento para pollos de engorda. Veterinária México, v.24, n.2, 1994.

BERTECHINI, .A.G.; HOSSAIN, S.M. Utilização de um tipo de probiótico como promotor de crescimento em rações de frangos de corte. In: CONFERÊNCIA APINCO DE CIÊNCIA e TECNOLOGIA AVÍCOLAS, Santos, 1993. Anais... Santos:APINCO, 1993.p.1.

CHOI, J.H.; RYU, K.S. Responses of broilers to dietary zinc bacitracin at two diferents planes of nutrition. British Poultry Science. v.28., p.113-118, 1987.

FERREIRA, D.F. Sistema de análise de Variância de Dados Balanceados. Pacote computacional, Dex - UFLA:Lavras, 1998. 
GARCIA, R.G.; ARIKI, J.; MORAES, V.M.B; MURATA, L.S.; KRONKA, S.N.; BORGES, S.A. Ação isolada ou combinada da apramicina e dos ácidos fórmico + propiônico no desempenho de frangos de corte. In: CONFERÊNCIA APINCO 1999 DE CIÊNCIA E TECNOLOGIA AVÍCOLA, Campinas, 1999. Trabalhos de pesquisa... Campinas:FACTA, 1999. p.36.

HENRIQUE, .A.P.F.; FARIA, D.E.; FRANZOLIN, R.; ITO, D.T. Uso de probióticos e antibióticos como promotores de crescimento para frangos de corte. Anais..., XXXV Reunião da SBZ, p.297-299, 1998.

IAFIGLIOLA, M.C. O uso de cobre e antibiótico como promotores de crescimento em rações para frangos de corte. Piracicaba, 1999. 68 p. Dissertação (mestrado em Zootecnia) - Curso de PósGraduação em Zootecnia, Universidade de São Paulo, 1999.

KRINKE, .A.L.; JAMROZ, D. Effects of feed antibiotic avoparcin on organ morphology in broiler chickens. Poultry Science., 75:705-710, 1996.

LODDI, M.M.; GONZALES, E.; TAKITA, T.S.; MENDES, .A.A.; ROÇA, R.O. Uso de probiótico e antibiótico sobre o desempenho, $o$ rendimento de carcaça e a qualidade de carcaça de frangos de corte. Revista Brasileira de Zootecnia, 29(4), 1121$1131,2000$.

PENSACK, J.M.; WANG, G.T.; SIMKINS, K.L. Avoparcin - a growth promoting feed antibiotic for broiler chickens. Poultry Science., v.61, p.1009-1012, 1982.

ROSTAGNO, H.S.; ALBINO, L.F.T.; DONZETE, J.L.; GOMES, P.C.; FERREIRA, .A.S.; OLIVEIRA, R.F.; LOPES, D.C.
Composição de alimentos e exigências nutricionais. (Tabelas Brasileiras). Viçosa: UFV, Imprensa Universitária, 2000, 141p.

SPRING, P. Yeast's secret weapon aids animal production. Anais do Simpósio sobre Aditivos Alternativos na Nutrição Animal. Campinas,SP p.41-50, 2000.

TEIXEIRA, A. S.; SANTOS, E. C.; FREITAS, R. T. F.; BERTECHINI, A. G.; RODRIGUES, P. B.; DIAS, E. S.; TORRES, D. M.; SANTOS, E. C.; SANTOS, . A. V.; GIACOMETTI, R. A. Efeito do período de fornecimento de antibióticos sobre o desempenho de frangos de corte. In: REUNIÃO ANUAL DA SOCIEDADE BRASILEIRA DE ZOOTECNIA, 2002, Recife. Anais... Recife: SBZ, 2002. 4 p. CD-ROM.

WOLKE, L.F.; FLEMING, J.S.; MIRA, R.T. et al. Utilização do probiótico Bacillus natto como promotor de crescimento na alimentação de frangos de corte. In: REUNIÃO ANUAL DA SOCIEDADE BRASILEIRA DE ZOOTECNIA, 33, 1996. Fortaleza. Anais..., Fortaleza:SBZ, p.36-38, 1996.

ZUANON, J.A.S.; FONSECA, J.B.; ROSTAGNO, H.S.; SILVA, M.A. Desempenho de frangos de corte alimentados com rações contendo antibióticos e probióticos adicionados isoladamente, associados ou em uso sequencial. Revista Brasileira de Zootecnia, 